\title{
Stability of pyrolysis oil-water emulsion
}

\section{Pipat Subsuksumran ${ }^{1, a}$, Prakorn Kittipoomwong ${ }^{1, b}$ Monpilai Narasingha ${ }^{1, c}$ and Wirachai Soontornrangson ${ }^{2, d}$}

\author{
${ }^{1}$ Chemical Engineering Department, Faculty of Engineering, King Mongkut's University of \\ Technology North Bangkok 1518 Pracharat 1 Rd. Bangsue Bangkok 10800 Thailand \\ ${ }^{2}$ Thailand Institute of Scientific and Technological Research (TISTR), Technopolis, \\ Klong5 Klong Luang Pathumthani 12120 Thailand \\ apipat.subsuksumran@gmail.com, bprakornk@kmutnb.ac.th, \\ cmhc@kmutnb.ac.th, dwirachai@tistr.com
}

Keywords: pyrolysis, plastic HDPE, emulsification, stability, phase separation, ultrasonic

\begin{abstract}
Emulsification of pyrolysis oil produced from plastic waste has been experimented. The employed cascade heating steps and heating rates pyrolysis process provides $80 \%$ product yield using waste plastic or recycles HDPE pellets as a raw material. Water-in-oil emulsion is produced ultrasonically and mechanically with Span 80 as a surfactant. The emulsion stability was assessed by water droplet size and visual observation of any phase separation. An ultrasonic mixer is found to be more effective than mechanical homogenizer in terms of homogenous stability to emulsify plastic oil with water. For emulsion with $10 \%$ water by volume, the emulsion is observed to be stable for up to 24 hours after mixing.
\end{abstract}

\section{Introduction}

Plastic grocery bags have several attractive attributes including low cost, light weight and sturdy which is resulted in ascending demands. The significant consumption of plastic bag requires an appropriate waste management. In Bangkok, Thailand more than 1,800 tons of plastic bag waste went to landfills each year which is unsustainable and could causes numerous environmental problems. A pyrolysis of waste plastic is an appropriate technique for both transforming and recycling of waste plastic [1]. The properties of plastic oil compared favorably to the requirement for diesel engine thus $100 \%$ waste plastic oil could be used as a diesel fuel-substitute [2]. However, there is an emission drawback from using plastic oil fuel as the larger amount of $\mathrm{NO}_{\mathrm{x}}, \mathrm{CO}$, and unburned hydrocarbon emission compared to that of diesel fuel have been reported [2,3]. The presence of alkenes group and nitrogen compound from fast pyrolysis process in plastic oils caused soot and nitrogen oxide, i.e. $\mathrm{NO}_{\mathrm{x}}$ when burnt $[1,4]$. Upgrading plastic oil through emulsification could bring a significant reduction in $\mathrm{NO}_{\mathrm{x}}, \mathrm{CO}$, and soot $[5,6]$.

One of the major challenges of plastic oil upgrading process is the emulsion stability. Small disperse phase droplet size is preferable due to less chance of droplets coalescence and phase seperation. In addition, a diesel combustion improvement is observed when emulsion with water droplet size of $0.53 \mu \mathrm{m}$ is employed compared to that of $5.5 \mu \mathrm{m}$ sample [7]. The increase of contact area between water and fuel for emulsion of smaller water droplet size enhances the heat and mass transfer between fuel and water. The proper choice of technique used to deform the dispersed phase into fine droplets method for emulsification can enhance emulsion stablity. The experimental observations in water-diesel mixture show a disperse phase droplet size produced by ultrasonic mixer was smaller than homogenizer $[5,6]$.

In this study, pylorysis of waste plastics and subsequent emulsification of pylorysis oil were investigated. An appropriate source of raw material for pylorysis oil production was examined in terms of elemental analysis and heating value measurement. Emulsifications of pyolysis were conducted to study the effects of several process variables including water content and mixing process. Two different emulsification methods, i.e. ultrasonic and mechanical emulsifications were compared in terms of stability, droplet size and rheological properties of emulsions to define an effective emulsification conditions. 


\section{Materials and method}

Materials: Plastic oils were produced from two different sources of raw material. The first source of was obtained by collecting plastic grocery bags from local retailers and represents a typical plastic waste. Recycled HDPE pellets were employed as an alternative source for a pyrolysis oil production.

Plastic oil preparation and characterization: Thermochemical conversions of HDPE into oils were conducted via a batch type pyrolyzer [8]. A 3-liter capacity of 316 stainless steel pyrolyzer is externally heated by an electric furnace with a cascaded temperature control as shown in Table 1. Industrial grade nitrogen $\left(\mathrm{N}_{2}\right)$ was fed into pyrolyzer for purging air from the system prior to the pyrolysis process. Plastic oil was obtained from a water-cool condenser maintained at about $15^{\circ} \mathrm{C}$ and was stored in a 2 liter water-cool glass bottle.

Table 1 The cascaded temperature control of pyrolysis process

\begin{tabular}{|c|c|}
\hline Temperature $\left({ }^{\circ} \mathrm{C}\right)$ & Time $(\mathrm{hr})$. \\
\hline $30-400$ & 0.37 \\
\hline $400-500$ & 1.00 \\
\hline 500 & 1.00 \\
\hline $500-600$ & 1.40 \\
\hline 600 & 1.00 \\
\hline
\end{tabular}

Chemical analysis of plastic oil was conducted via a Leco truspec CHNS elemental analyzer according to ASTM D5373 and ASTM D4239. In addition, the gross calorific value was measrued by Parr 6300 Calorimeter according to ASTM D5865.

Emulsion preparation and characterization: A water-in-oil emulsion was produced with a commercial grade of Span80 (Sorbitan monooleate) as a surfactant. For the dispersed phase, the amount distillated water was varied between $10-30 \%$ by volume. The surfactant concentration in the entire volume of emulsions was kept at $2 \%$ by volume with plastic oil occupied the rest. The emulsions were mixed at room temperature by either a) homogenizer (IKA-Ultra-Turrex T50) operated at 4,000 rpm for $15 \mathrm{~min}$ or b) ultrasonic mixer (Bandelin Sonopuls) operated at $20 \mathrm{kHz}$, for $15 \mathrm{~min}$.

The visual characterizations of emulsion were assessed in term of average size of water droplet and static stability test. A micrograph of emulsion was taken by microscope (Olympus,IX-83ZDC) and subsequently analyzed by image analysis software (ImageJ ver. 1.47) to obtain an average size of water droplet. After mixing, samples were placed in $80 \mathrm{ml}$ graduated cylinder to record the levels of interphase against time. A phase separation (if any) between clear oily phase and milky emulsion phase was observed at a periodic interval [9]. In addition, rheological characterizations of emulsions were assessed in term of kinematic viscosities measured by Ubbelohde Viscometer. In case of a viscous sample, apparent viscosities were also measured by MV-2000 series II Cannon ${ }^{\circledR}$ Rotary Viscometer with shear rate varied from 10-100 rpm corresponding to 12.24-122.4 s-1.

\section{Results and Discussion}

The elemental and gross calorific value of plastic oil made from grocery bag and that made from recycled HDPE pellets are shown in Table 2. The elemental analysis results revealed no significant difference in CHNS composition of plastic oils made from grocery bags and recycled HDPE pellets. In addition, the gross calorific values of both sources are of similar value according to calorimeter measurement and empirical correlation given by Jian and Ellis [10]. As a result, grocery bags can be reasonably substituted by recycled HPDE pellets. Consequently, the HDPE pellets are used as a primary raw material source. 
Table 2 Elemental analysis and gross calorific value of plastic oils made from different sources

\begin{tabular}{|c|c|c|c|c|c|c|}
\hline \multirow{2}{*}{ Source } & \multicolumn{3}{|c|}{ Element analysis (\% mass of element) } & \multicolumn{2}{c|}{$\begin{array}{c}\text { Gross calorific value } \\
\text { (MJ/kg) }\end{array}$} \\
\cline { 2 - 7 } & $\mathrm{C}$ & $\mathrm{H}$ & $\mathrm{N}$ & $\mathrm{S}$ & Calorimeter & Correlation \\
\hline Grocery bags & 85.15 & 13.45 & 0.20 & 0.21 & 45.85 & 48.02 \\
\hline $\begin{array}{c}\text { Recycled HDPE } \\
\text { pellets }\end{array}$ & 83.97 & 13.46 & 0.13 & 0.14 & 43.44 & 47.40 \\
\hline
\end{tabular}

Emulsification mixing process gives rise to variations in the emulsion stability. The homogenous emulsion is observed as milky emulsion initially after mixing. As for the homogenizer case, two distinguishable zones were developed after settling for a few minutes. The volume of top yellowish clear layer continues to increase with time at the expense of lower milky emulsion. The top layer has a viscosity of about $2.8 \mathrm{~mm}^{2} / \mathrm{s}$ which is closed to that of plastic oil i.e. $2 \mathrm{~mm}^{2} / \mathrm{s}$. As for the ultrasonic case, there is also an appearance of thin clear layer. The observation of $10 \%$ water emulsion showed that the height of top layer produced ultrasonically after $24 \mathrm{hrs}$ is $0.3 \mathrm{~cm}$ which is significantly less than $11 \mathrm{~cm}$ measured in homogenizer case. The stability of emulsion is characterized by a stability parameter (S) defined as the height of emulsion after settling for $24 \mathrm{hrs}$. to total height (volume) of water-oil mixture. In Fig. 1 (a), the stability parameter of emulsion with 10\% volume of distillated water is plotted as a function of time for ultrasonic and homogenized mixing. The dramatic difference in stability trend between ultrasonic mixer and homogenizer supports the previous discussion.

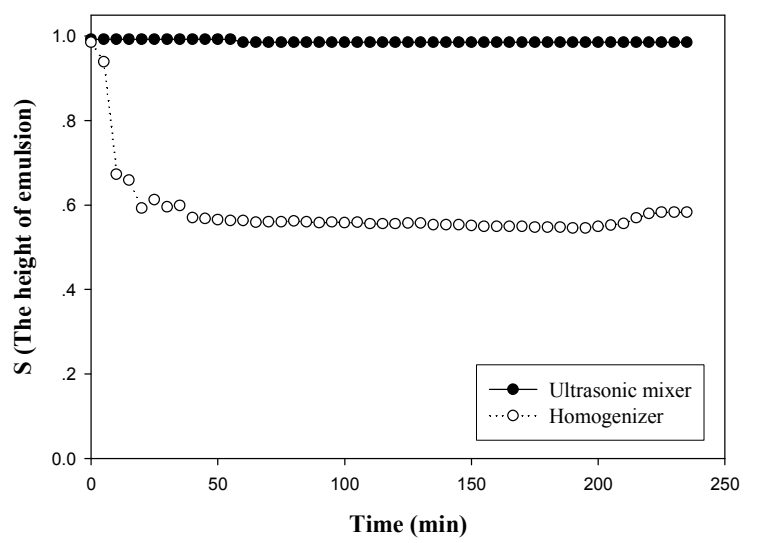

(a)

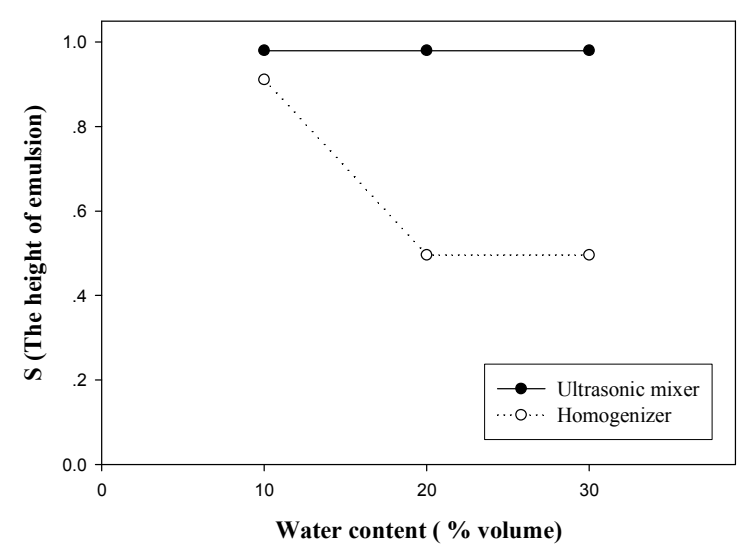

(b)

Fig. 1 Stability parameter of emulsion as a function of (a) time (b) water content

Water content is another important consideration. The stability parameter of emulsion plotted as a function of water content is shown in Fig. 1 (b). The stability parameter of both ultrasonic and homogenizer decreases as water content increased. This is to be expected due to the constant surfactant concentration leading to less stability as water fraction increases. Viscosity of emulsion was found to increase with water content in a non-linear fashion.

The size of water droplet is evaluated of whether it is changed into microstructure. The micrograph of 9:1 volume ratio with surfactant concentration of $2 \%$ by volume, oil-water mixture is shown in Fig. 2. The average water droplet size of $0.6 \mu \mathrm{m}$ prepared by ultrasonic mixer is smaller than those of the emulsion formed by homogenizer, i.e. $1.11 \mu \mathrm{m}$. This is comparable to the size of water droplet in diesel emulsion reported elsewhere [4]. Smaller water droplet size produced by ultrasonic mixer leads to the reduction of contact surface area between droplet and consequently more stability due to less potential of droplet coalescence. 

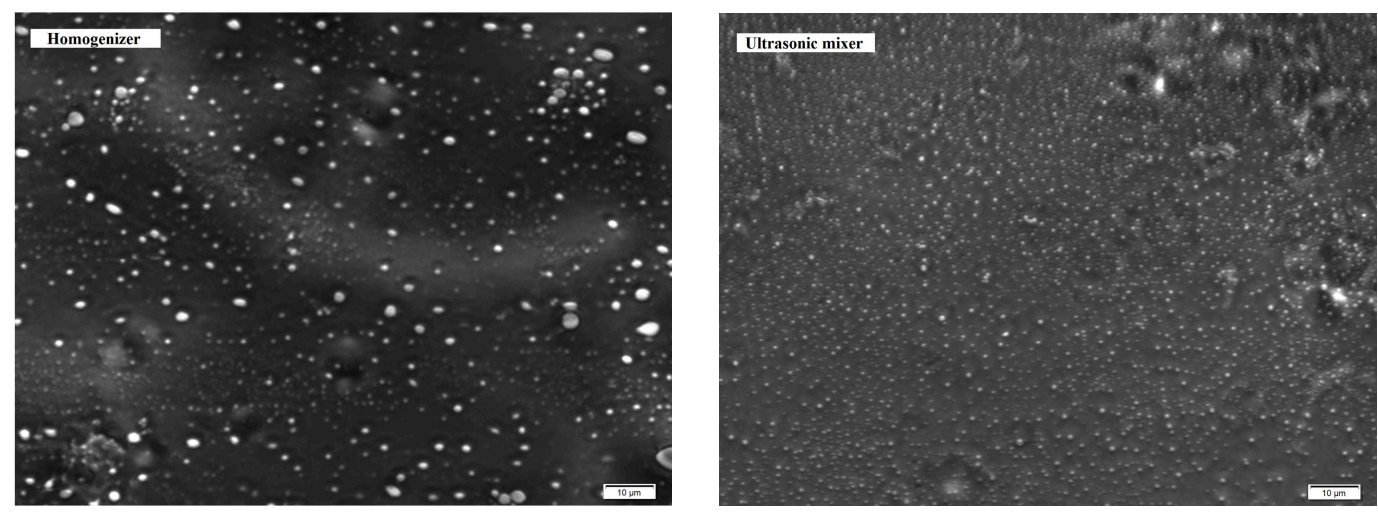

Fig. 2 Optical microscopy photograph of water droplets dispersion

\section{Summary}

The emulsion of water and plastic oil produced by ultrasonic mixing appears to be largely homogenous over 24 hours observation period. An alternative emulsion produced by mechanical homogenizer exhibits phase separation over the same condition. The water droplets measurement also show smaller average water droplet for ultrasonic mixer. The stability varied linearly with water content.

\section{Acknowledgements}

This work was financially supported by Thailand Institute of Scientific and Technological Research (TISTR) under the Associate Graduated Program. The authors are thankful to Mr. Hiran Jinda, Ms. Prachtida Pornprasert, and Ms. Jissharaporn Kittivittayarat for their assistance.

\section{References}

[1] Brajendra K.Sharma, Bryan R. Moser, Karl E.Vermillion, Kenneth M.Doll, and Nandakishore Rajagopalan: Production, characterization and fuel properties of alternative diesel fuel from pyrolysis of waste plastic grocery bags. Fuel Process Technol. Vol.122 (2014), p.79-90

[2] M. Mani, C. Subash, and G. Nagarajan: Performance, emission and combustion characterization of a DI diesel engine using waste plastic oil. Appl.Therm.Eng. Vol.29 (2009), p.2738-2744

[3] Yocai Liang, Gequn Shu, Haiqiao Wei, and Wei Zhang: Effect of oxygen enriched combustion and water-diesel engine. Energ.Convers.Manage. Vol.73 (2013), p.69-77

[4] A. Abouljas, T. Makayssi, L. Bilali, K. El harfi, M. Nadifiyine, and M. Benchanaa: Co-pyrolysis of oil shale and High density polyethylene: Structural characterization of the oil. Fuel Process. Technol. Vol.96 (2012), p.203-208

[5] Y. Kitamura, Q. Huang, Y. Oka, and T. Takahashi: Flashing of superheated water in oil emulsions - microexplosion of emulsified fuel. J.Chem.Eng.Jpn. Vol.23 (1990), p.711-715

[6] E. Mura, P. Massoli, C. Josset, K. Loubar, and J. Bellettre: Study of the micro-explosin temperature of water in oil emulsion droplets during the Leidenfrost effect. Exp.Therm.Fluid Sci. Vol.43 (2012), p.63-70

[7] A. Attia and A. R. Kulchitskiy: Influence of the structure of water-in-fuel emission on diesel engine performance. Fuel Vol.116 (2014), p. 703-708

[8] Khanet Thepoharak, Kraipat Cheenkachorn, and Thanes Utistham: Pyrolysis of waste lubricating oil. Master Thesis, King Mongkut's University of Technology North Bangkok (2012)

[9] N. Ongsirimongkol and M. H. Narasingha: Effects of Stabilizing Agents on Stability and Rheological Characteristics of the Highly-Loaded Coal-Water Slurry. International Journal of Chemical Engineering and Applications Vol. 3 (2012), p.49-52

[10] Xiaoxiang Jian and Naoko Ellis: Upgrading Bio-oil through Emulsification with Biodiesel: Mixture Production, Energy Fuels Vol. 24 (2010) , p. 1358-1364 